\title{
Solution Algorithms for Single-Machine Group Scheduling with Learning Effect and Convex Resource Allocation
}

\author{
Wanlei Wang $\left(\mathbb{D},{ }^{1}\right.$ Jian-Jun Wang, ${ }^{2}$ and Ji-Bo Wang ${ }^{3}$ \\ ${ }^{1}$ College of Mechanical and Electronic Engineering, Dalian Minzu University, Dalian 116600, China \\ ${ }^{2}$ School of Economics and Management, Dalian University of Technology, Dalian 116024, China \\ ${ }^{3}$ School of Science, Shenyang Aerospace University, Shenyang 110136, China \\ Correspondence should be addressed to Wanlei Wang; wwl@dlnu.edu.cn
}

Received 22 December 2020; Revised 19 January 2021; Accepted 10 February 2021; Published 3 March 2021

Academic Editor: Lei Xie

Copyright (c) 2021 Wanlei Wang et al. This is an open access article distributed under the Creative Commons Attribution License, which permits unrestricted use, distribution, and reproduction in any medium, provided the original work is properly cited.

This paper deals with a single-machine resource allocation scheduling problem with learning effect and group technology. Under slack due-date assignment, our objective is to determine the optimal sequence of jobs and groups, optimal due-date assignment, and optimal resource allocation such that the weighted sum of earliness and tardiness penalties, common flow allowances, and resource consumption cost is minimized. For three special cases, it is proved that the problem can be solved in polynomial time. To solve the general case of problem, the heuristic, tabu search, and branch-and-bound algorithms are proposed.

\section{Introduction}

In the conventional scheduling models and problems, it is generally assumed that the job processing times are constants, but in practice, examples can be found to illustrate that the job processing times are not necessarily constants (Shabtay and Steiner [1], Biskup [2], and Azzouz et al. [3]). More recently, Zhu et al. [4] considered resource allocation single-machine scheduling problems with learning effects and group technology. For the linear and convex resource allocation models, they proved that problem of minimizing the weighted sum of makespan and total resource cost can be solved in polynomial time. Lu et al. [5] revisited the same model with Zhu et al. [4], but they considered the case of resource availability is limited. For the makespan minimization subject to limited resource availability, they proposed heuristic and branch-and-bound algorithms. Sun et al. [6] and Lv et al. [7] worked on singlemachine scheduling group problems with learning effects and resource allocation. Under the slack (SLK) due-date assignment, for the linear weighted sum of scheduling cost and resource consumption cost minimization, Sun et al. [6] proved that the problem can be solved in polynomial time. However, Lv et al. [7] showed that the results of Sun et al. [6] are incorrect by two counter-examples, and Lv et al. [7] also provided the corrected results under a special case. In this paper, we will consider the same model with Sun et al. [6] and Lv et al. [7], i.e., three popular features in the recent years: group technology, resource allocation, and learning effect. The contributions of this study are given as follows: (1) we study the SLK assignment single-machine group scheduling problem along with learning effect and convex resource allocation; (2) the optimal properties are provided for the total cost (including earliness, tardiness, common flow allowances, and resource consumption cost) minimization; (3) we propose the heuristic, tabu search, and branch-and-bound algorithms to solve the problem.

The reminder of this paper is organized as follows. In Section 2, the relevant literature review is presented. The problem statement is presented in Section 3. Section 4 gives some properties of the problem. In Section 5, some special cases are discussed. In Section 6, for the general case, solution algorithms are proposed. Finally, the conclusions are given in Section 7.

\section{Literature Review}

In this section, we restrict our literature review to papers that study scheduling problems with learning effects, resource allocation, and/or group technology. 
In manufacturing environments, after learning, the time required for workers (machines) to process some jobs is decreasing, which causes scheduling problems with learning effects (Biskup [2]). Wang et al. [8] considered the flow shop problem with a learning effect. Under two-machine and release dates, the goal is to minimize the weighted sum of makespan and total completion time. They proposed a branch-and-bound algorithm and a multiobjective memetic algorithm to solve the problem. Wang et al. [9] considered flow shop problems with truncated learning effects. For the makespan and total weighted completion time minimizations, they proposed heuristics and branch-and-bound algorithms. Yan et al. [10] and Wang et al. [11] studied single-machine scheduling problems with learning effects and release times. Sun et al. [12] investigated flow shop problem with general position weighted learning effects. For the total weighted completion time minimization, they proposed some heuristics and a branch-and-bound algorithm to solve the problem.

In addition, scheduling problems with resource allocation (controllable processing times) have also attracted considerable interest from researchers (Shabtay and Steiner [1]), that is, the scheduler can control processing times of jobs by allocating a common continuously nonrenewable resource. Kayvanfar et al. [13, 14] considered single-machine scheduling with controllable processing times. For the total tardiness and earliness minimization, Kayvanfar et al. [13] proposed a mathematical model and three heuristic techniques; Kayvanfar et al. [14] proposed a drastic hybrid heuristic algorithm. Lu and Liu [15] considered single machine scheduling problems with resource allocation and position-dependent workloads. They proposed a bicriteria analysis for the scheduling cost and total resource consumption cost. Tsao et al. [16] considered energy-efficient single-machine scheduling problem with controllable processing time. Under differential electricity pricing, they proposed a mixed-integer programming model and a fuzzy control approach. Mor et al. [17] considered single-machine scheduling problems with resource-dependent processing times. For a large set of that the scheduling criterion can be represented as one that includes positional penalties; they proposed heuristic algorithms to solve the problems. Kayvanfar et al. [18] considered unrelated parallel machines scheduling problem with controllable processing times. For the linear weighted sum of tardiness, earliness, jobs compressing and expanding costs, and makespan minimization, they proposed a mixed integer programming model and some heuristics. Zarandi and Kayvanfar [19] considered a biobjective identical parallel machines scheduling problem with controllable processing times. The goal is to simultaneously minimize total cost of tardiness and earliness as well as compression and expansion costs of job processing times and makespan. They proposed two multiobjective evolutionary algorithms to solve the biobjective problem. Kayvanfar et al. [20] studied identical parallel machine scheduling problem with controllable processing times. For the linear weighted sum of tardiness, earliness, and job compressions/expansion cost minimization, they proposed a mixed integer linear programming model and some heuristic algorithms to solve the problem.
A third possible aspect is that scheduling problems with group technology (GT, see studies by Mosier et al. [21] and Webster and Baker [22]), that is, GT is an approach to manufacturing that seeks to improve efficiency in high-volume production by exploiting the similarities of different products and activities in their production (see studies by Yang and Yang [23] and Ji et al. [24]). Xu et al. [25] and Liu et al. [26] considered single-machine group scheduling with deteriorating jobs and ready times. For the makespan minimization, $\mathrm{Xu}$ et al. [25] proved that some special cases can be solved in polynomial time; for the general case, Liu et al. [26] proposed a branch-and-bound algorithm. Li and Zhao [27] considered single machine scheduling problem with group technology. Under multiple due windows assignment, they proved that the total cost (including earliness, tardiness, and due windows) minimization can be solved in polynomial time. Zhang and Xie [28] studied the single machine scheduling problem with position dependent processing times. Under group technology and ready times, for the makespan minimization, they proved that a special case can be solved in polynomial time. Ji and $\mathrm{Li}$ [29] studied single-machine group scheduling with variable job processing times (including resource allocation, learning effects, and deteriorating jobs). They proved that two versions of problem can be solved in polynomial time. Zhang et al. [30] considered single-machine group scheduling problems with position-dependent processing times. They proved that the makespan and the total completion time minimization can be solved in polynomial-time algorithm, respectively. Muştu, and Eren [31]) studied the single-machine scheduling problem with sequence-independent setup times and time-dependent learning and forgetting effects. They proved that the makespan minimization is ordinary NP-hard, and they also proposed an integer nonlinear programming and a dynamic programming to solve the problem. Extensive surveys of different scheduling models and problems with group technology can be found in studies by Allahverdi [32] and Neufeld et al. [33].

More recently, Wang et al. [34] and Lu et al. [35] delved into single-machine resource allocation scheduling problems with learning effects. He et al. [36] considered the singlemachine resource allocation scheduling with truncated jobdependent learning effect. Under linear and convex resource allocations, polynomial time algorithms are developed to solve the problem. Li et al. [37] considered single-machine scheduling with general job-dependent learning curves and controllable processing times. They proved that some regular and nonregular objective minimizations can be solved in polynomial time. Wang et al. [38] considered single-machine scheduling with truncated learning effects and resource allocation. For total weighted flow time cost and total resource consumption cost, they provided a bicriteria analysis. Geng et al. [39] and Sun et al. [40] investigated two-machine no-wait flow shop scheduling with resource allocation and learning effect. For the common due date assignment, Geng et al. [39] proved that two versions of the scheduling criteria and resource consumption cost can be solved in polynomial time. For the slack due-date assignment, Sun et al. [40] proved that three versions of the scheduling criteria and resource consumption cost can be solved in polynomial time. Liu and Jiang [41] explored due-date assignment scheduling problems with job- 
dependent learning effects and convex resource allocation. Zhang et al. [30] considered single-machine group scheduling problems with position-dependent learning effects. They proved that the makespan and total completion time minimizations can be solved in polynomial time algorithm. Wang and Liang [42] and Liang et al. [43] investigated single-machine resource allocation scheduling with deteriorating jobs and group technology. Liao et al. [44] studied a two-competing group parallel machines scheduling problem with truncated job-dependent learning effects. Under serial-batching machines, for the makespan minimization, they proposed a greedy algorithm.

To the best of our knowledge, apart from the recent papers of Sun et al. [6] and Lv et al. [7], the single-machine slack duedate assignment scheduling problem with resource allocation, group technology, and learning effects has not been investigated. In this paper, we consider the same model as in Sun et al. [6] and Lv et al. [7], but with the tabu search and branch-andbound algorithms to solve the problem.

\section{Problem Formulation}

We have $n$ jobs grouped into $f$ groups (i.e., $\left.\left(G_{1}, G_{2}, \ldots, G_{f}\right)\right)$ to be processed by a single machine, where the number of jobs in the group $G_{i}(i=1,2, \ldots, f)$ is $m_{i}$, i.e., $\sum_{i=1}^{f} m_{i}=n$. Each group $G_{i}(i=1,2, \ldots, f)$ has an independent setup time $s_{i}$ and contains $m_{i}$ jobs which are processed consecutively. Let $J_{i, j}$ be the $j$ th job in group $G_{i}$, $i=1,2, \ldots, f, j=1,2, \ldots, m_{i}$, i.e., group $G_{i}$ has jobs $J_{i, 1}, J_{i, 2}, \ldots, J_{i, m_{i}}$. Let $J_{[i],[j]}$ be the job in the $i$ th group position and $j$ th internal job position. As in the study by Liang et al. [43], if the job $J_{i, j}$ is scheduled in $r$ th position in group $G_{i}$, the actual processing time of job $J_{i, j}$ is

$$
p_{i, j}^{A}=\left(\frac{p_{i, j}{ }^{a_{i, j}}}{u_{i, j}}\right)^{\eta}
$$

where $\eta$ is a constant positive parameter, $p_{i, j}$ is the normal processing time of job $J_{i, j}, a_{i, j} \leq 0$ is the learning rate (see the study by Biskup [2]) of job $J_{i, j}, u_{i, j} \geq u_{i}^{\prime}>0, u_{i}^{\prime}$ is the minimal resource allocation to the jobs of group $G_{i}$ (if $u_{i, j}$ is close to zero, $p_{i, j}^{A}$ will be close to infinity, which is not realistic; hence, we set $\left.u_{i, j} \geq u_{i}^{\prime}>0\right)$. Let $C_{i, j}\left(d_{i, j}\right)$ be the completion time (duedate) of job $J_{i, j}$. For the slack (SLK) due-date assignment, we assume that the due-date of job $J_{i, j}$ is $d_{i, j}=p_{i, j}^{A}+q_{i}$, where $q_{i}$ is the common flow allowance for group $G_{i}$ and $q_{i}$ is a decision variable. Let $E_{i, j}=\max \left\{d_{i, j}-C_{i, j}, 0\right\} \quad\left(T_{i, j}=\max \left\{C_{i, j}-d_{i, j}\right.\right.$, $0\}$ ) be the earliness (tardiness) of job $J_{i, j}$; our goal is to find the optimal group sequence $\pi_{G}^{*}$, job sequence $\pi_{i}^{*}(i=1,2, \ldots, f)$ within group $G_{i}$, and resource allocation such that the following cost is minimized:

$$
\sum_{i=1}^{f} \sum_{j=1}^{m_{i}}\left(\alpha_{i} E_{i, j}+\beta_{i} T_{i, j}+\gamma_{i} q_{i}\right)+\sum_{i=1}^{f} \sum_{j=1}^{m_{i}} v_{i, j} u_{i, j},
$$

where $\alpha_{i}, \beta_{i}$, and $\gamma_{i}$ are the nonnegative parameters of group $G_{i}$ and $v_{i, j}$ represents the per unit cost of the resource $u_{i, j}$ allocated to job $J_{i, j}$. Using the three-field classification (see the studies by Shabtay and Steiner [1], Biskup [2], and Azzouz et al. [3]), the problem can be denoted as

$$
1\left|\mathrm{GT}, s_{i}, \mathrm{CRA}, \mathrm{SLK}\right| \sum_{i=1}^{f} \sum_{j=1}^{m_{i}}\left(\alpha_{i} E_{i, j}+\beta_{i} T_{i, j}+\gamma_{i} q_{i}\right)+\sum_{i=1}^{f} \sum_{j=1}^{m_{i}} v_{i, j} u_{i, j} \text {, }
$$

where GT denotes the group technology and CRA represents the convex function of resource allocation (1).

\section{Some Properties}

Obviously, an optimal sequence exists that starts at time zero and without any machine idle time between all the jobs. Similar to the study by Adamopoulos and Pappis [45], we have the following.

Lemma 1. If $C_{[i],[j]} \leq d_{[i],[j]} \Longrightarrow C_{[i],[j-1]} \leq d_{[i],[j-1]},(i=1,2$, $\left.\ldots, f ; j=1,2, \ldots, m_{i}\right)$.

If $C_{[i],[j]} \geq d_{[i],[j]} \Longrightarrow C_{[i],[j+1]} \geq d_{[i],[j+1]}, \quad(i=1,2, \ldots$, $f ; j=1,2, \ldots, m_{i}$ ).

Lemma 2. For the problem $1\left|G T, s_{i}, C R A, S L K\right| \sum_{i=1}^{f} \sum_{j=1}^{m_{i}}$ $\left(\alpha_{i} E_{i, j}+\beta_{i} T_{i, j}+\gamma_{i} q_{i}\right)+\sum_{i=1}^{f} \sum_{j=1}^{m_{i}} v_{i, j} u_{i, j}$, there exists an optimal schedule such that the optimal value of $q_{[i]}$ coincides with the job completion time of the group $G_{[i]}$, i.e., $q_{[i]}=C_{[i],\left[k_{i}-1\right]}=S_{[i]}+s_{[i]}+\sum_{j=1}^{k_{i}-1} p_{[i],[j]}^{A}$, where

$$
k_{[i]}=\min \left\{m_{[i]}, \max \left\{0,\left\lceil\frac{m_{[i]}\left(\beta_{[i]}-\gamma_{[i]}\right)}{\alpha_{[i]}+\beta_{[i]}}\right\rceil\right\}\right\},
$$

and $S_{[i]}$ is the starting time of group $G_{[i]}$.

Lemma 3 (see [43]). For a given sequence $\pi$, the optimal resource allocation is

$$
u_{[i],[j]}^{*}=\max \left[u_{[i],[j]}, u_{[i]}^{\prime}\right],
$$

where

$$
u_{[i],[j]}= \begin{cases}\left(\frac{\eta\left(\alpha_{[i]} j+\sum_{h=i}^{f} m_{[h]} \gamma_{[h]}\right)}{v_{[i],[j]}}\right)^{(1 /(\eta+1))}\left(p_{[i],[j]} j^{a_{[i],[j]}}\right)^{(\eta /(\eta+1))}, & i=1, \ldots, f ; j=1, \ldots, k_{[i]-1}, \\ \left(\frac{\eta \beta_{[i]}\left(m_{[i]}-j\right)}{v_{[i],[j]}}\right)^{(1 /(\eta+1))}\left(p_{[i],[j]} j^{a_{[i],[j]}}\right)^{(\eta /(\eta+1))}, & i=1, \ldots, f ; j=k_{[i]}, \ldots, m_{[i]} .\end{cases}
$$


From the study by Liang et al. [43], we have

$$
\begin{aligned}
\sum_{i=1}^{f} & \sum_{j=1}^{m_{i}}\left(\alpha_{i} E_{i, j}+\beta_{i} T_{i, j}+\gamma_{i} q_{i}\right)+\sum_{i=1}^{f} \sum_{j=1}^{m_{i}} v_{i, j} u_{i, j} \\
= & \sum_{i=1}^{f}\left(\sum_{h=i}^{f} m_{[h]} \gamma_{[h]}\right) s_{[i]} \\
& +\left(\eta^{(1 /(\eta+1))}+\eta^{(-\eta /(\eta+1))}\right) \sum_{i=1}^{f} \sum_{j=1}^{k_{[i]}^{-1}}\left(\alpha_{[i]} j+\sum_{h=i}^{f} m_{[h]} \gamma_{[h]}\right)^{(1 /(\eta+1))}\left(\nu_{[i],[j]} p_{[i],[j]} j^{a_{[i],[j]}}\right)^{(\eta /(\eta+1))} \\
& +\left(\eta^{(1 /(\eta+1))}+\eta^{(-\eta /(\eta+1))}\right) \sum_{i=1}^{f} \sum_{j=k_{[i]}}^{m_{[i]}}\left(\beta_{[i]}\left(m_{[i]}-j\right)\right)^{(1 /(\eta+1))}\left(\nu_{[i],[j]} p_{[i],[j]} j^{a_{[i],[j]}}\right)^{(\eta /(\eta+1))} .
\end{aligned}
$$

Lemma 4 (see [43]). For the problem $1\left|G T, s_{i}, C R A, S L K\right|$ $\sum_{i=1}^{f} \sum_{j=1}^{m_{i}}\left(\alpha_{i} E_{i}, j+\beta_{i} T_{i, j}+\gamma_{i} q_{i}\right)+\sum_{i=1}^{f} \sum_{j=1}^{m_{i}} v_{i, j} u_{i, j}$, if the sequence of groups is given by $\pi_{G}=\left[G_{[1]}, G_{[2]}, \ldots, G_{[f]}\right]$, the optimal job sequence in the group $G_{[i]}(i=1,2, \ldots, f)$ can be obtained by the following assignment problem (AP-i):

$$
(\mathbf{A P}-\mathbf{i}) \operatorname{Min} \sum_{j=1}^{m_{[i]}} \sum_{h=1}^{m_{[i]}} \vartheta_{[i], j, h} x_{[i], j, h},
$$

$$
\begin{gathered}
\text { s.t. } \sum_{h=1}^{m_{[i]}} x_{[i], j, h}=1, \quad i=1,2, \ldots, f ; j=1,2, \ldots, m_{[i]}, \\
\sum_{j=1}^{m_{[i]}} x_{[i], j, h}=1, \quad i=1,2, \ldots, f ; h=1,2, \ldots, m_{[i]}, \\
x_{[i], j, h}=0 \text { or } 1,
\end{gathered}
$$

where

$$
\vartheta_{[i], j, h}=\left\{\begin{array}{l}
\left(\eta^{(1 /(\eta+1))}+\eta^{(-\eta /(\eta+1))}\right)\left(\alpha_{[i]} h+\sum_{l=i}^{f} m_{[l]} \gamma_{[l]}\right)^{(1 /(\eta+1))}\left(v_{[i], j} p_{[i], j} h^{a_{[i] j}}\right)^{(\eta /(\eta+1))}, \quad i=1,2, \ldots, f ; j=1,2, \ldots, m_{[i]} ; h=1,2, \ldots, k_{[i]}-1 \\
\left(\eta^{(1 /(\eta+1))}+\eta^{(-\eta /(\eta+1))}\right)\left(\beta_{[i]}\left(m_{[i]}-h\right)\right)^{(1 /(\eta+1))}\left(v_{[i], j} p_{[i], j} h^{a_{[i], j}}\right)^{(\eta /(\eta+1))}, \quad i=1,2, \ldots, f ; j=1,2, \ldots, m_{[i]} ; h=k_{[i]}, k_{[i]}+1, \ldots, m_{[i]} .
\end{array}\right.
$$

Lemma 5. The term $\sum_{i=1}^{f}\left(\sum_{h=i}^{f} m_{[h]} \gamma_{[h]}\right) s_{[i]}$ is minimized if $s_{[1]} \leq s_{[2]} \leq \cdots \leq s_{[f]}$

Proof. It is similar to the proof of Liang et al. [43].
Lemma 6. If the optimal job sequence within each group is given, the term,

$$
\begin{aligned}
& \left(\eta^{(1 /(\eta+1))}+\eta^{(-\eta /(\eta+1))}\right) \sum_{i=1}^{f} \sum_{j=1}^{k_{[i]}-1}\left(\alpha_{[i]} j+\sum_{h=i}^{f} m_{[h]} \gamma_{[h]}\right)^{(1 /(\eta+1))}\left(\nu_{[i],[j]} p_{[i],[j]} j^{a_{[i],[j]}}\right)^{(\eta /(\eta+1))} \\
& +\left(\eta^{(1 /(\eta+1))}+\eta^{(-\eta /(\eta+1))}\right) \sum_{i=1}^{f} \sum_{j=k_{[i]}}^{m_{[i]}}\left(\beta_{[i]}\left(m_{[i]}-j\right)\right)^{(1 /(\eta+1))}\left(\nu_{[i],[j]} p_{[i],[j]} j^{\left.a_{[i],[j]}\right)^{(\eta /(\eta+1))}}\right.
\end{aligned}
$$


is minimized if $m_{[1]} \gamma_{[1]} \geq m_{[2]} \gamma_{[2]} \geq \cdots \geq m_{[f]} \gamma_{[f]}$.

Proof. By using simple group interchanging technique, the result can be easily obtained.

\section{Polynomial Time Solvable Cases}

5.1. Case 1. As in the study by Liao et al. [44], if the groups have agreeable conditions, i.e., if $s_{i} \leq s_{h}$ implies $m_{i} \gamma_{i} \geq m_{h} \gamma_{h}$ for all groups $G_{i}$ and $G_{h}$, the problem $1 \mid \mathrm{GT}, s_{i}$, CRA, SLK, $\left(s_{i} \leq s_{h}\right) \Longrightarrow\left(m_{i} \gamma_{i} \geq m_{h} \gamma_{h}\right) \mid \sum_{i=1}^{f} \sum_{j=1}^{m_{i}}\left(\alpha_{i} E_{i, j}+\beta_{i} T_{i, j}+\gamma_{i} q_{i}\right)+$ $\sum_{i=1}^{f} \sum_{j=1}^{m_{i}} v_{i, j} u_{i, j}$ can be solved in polynomial time.

Lemma 7 (see [44]). For the problem $1 \mid G T, s_{i}, C R A, S L K$, $\left(s_{i} \leq s_{h}\right) \Longrightarrow\left(m_{i} \gamma_{i} \geq m_{h} \gamma_{h}\right) \mid \sum_{i=1}^{f} \sum_{j=1}^{m_{i}}\left(\alpha_{i} E_{i, j}+\beta_{i} T_{i, j}+\gamma_{i} q_{i}\right)$ $+\sum_{i=1}^{f} \sum_{j=1}^{m_{i}} v_{i, j} u_{i, j}$, if the groups have agreeable conditions, i.e., if $s_{i} \leq s_{h}$ implies $m_{i} \gamma_{i} \geq m_{h} \gamma_{h}$ for all groups $G_{i}$ and $G_{h}$, then the optimal group sequence $\pi_{G}^{*}$ can be obtained by sequencing groups in nondecreasing order of $s_{i}$, or equivalently, the optimal group sequence $\pi_{G}^{*}$ can be obtained by sequencing groups in nonincreasing order of $m_{i} \gamma_{i}$.
For the problem $1 \mid \mathrm{GT}, s_{i}$, Con, $\left(s_{i} \leq s_{h}\right) \Longrightarrow\left(m_{i} \gamma_{i} \geq m_{h}\right.$ $\left.\gamma_{h}\right) \mid \sum_{i=1}^{f} \sum_{j=1}^{m_{i}}\left(\alpha_{i} E_{i, j}+\beta_{i} T_{i, j}+\gamma_{i} q_{i}\right)+\sum_{i=1}^{f} \sum_{j=1}^{m_{i}} v_{i, j} u_{i, j}$, the optimal solution algorithm is given as follows:

Theorem 1 (see [44]). The problem,

$$
\begin{gathered}
\text { 1|GT, } s_{i}, \text { CRA, SLK, }\left(s_{i} \leq s_{h}\right) \Longrightarrow\left(m_{i} \gamma_{i} \geq m_{h} \gamma_{h}\right) \mid \\
\sum_{i=1}^{f} \sum_{j=1}^{m_{i}}\left(\alpha_{i} E_{i, j}+\beta_{i} T_{i, j}+\gamma_{i} q_{i}\right)+\sum_{i=1}^{f} \sum_{j=1}^{m_{i}} v_{i, j} u_{i, j},
\end{gathered}
$$

can be solved by Algorithm 1 in $O\left(n^{3}\right)$ time.

5.2. Case 2. In this subsection, a special case will be considered, i.e., if $s_{i}=s, m_{i} \gamma_{i}=\overline{m \gamma}$, for $i=1,2, \ldots f$.

Lemma 8. For the problem $1 \mid G T, s_{i}, C R A, S L K, s_{i}=s, m_{i} \gamma_{i}=$ $\overline{m \gamma} \mid \sum_{i=1}^{f} \quad \sum_{j=1}^{m_{i}}\left(\alpha_{i} E_{i, j}+\beta_{i} T_{i, j}+\gamma_{i} q_{i}\right)+\sum_{i=1}^{f} \sum_{j=1}^{m_{i}} v_{i, j} u_{i, j}$, the optimal group sequence $\pi_{G}^{*}$ can be obtained by an assignment problem.

Proof. From Lemma 4, the sequence $\pi_{i}^{*}(i=1,2, \ldots, f)$ within the group $G_{i}$ can be obtained. For the group $G_{i}$, if $s_{i}=s$, and $m_{i} \gamma_{i}=\overline{m \gamma}(i=1,2, \ldots, f)$, from (7), we have

$$
\begin{aligned}
& \sum_{i=1}^{f} \sum_{j=1}^{m_{i}}\left(\alpha_{i} E_{i, j}+\beta_{i} T_{i, j}+\gamma_{i} q_{i}\right)+\sum_{i=1}^{f} \sum_{j=1}^{m_{i}} \nu_{i, j} u_{i, j} \\
& =\frac{f(f+1) \bar{s} \bar{\gamma}}{2}+\left(\eta^{(1 /(\eta+1))}+\eta^{(-\eta /(\eta+1))}\right) \sum_{i=1}^{f} \sum_{j=1}^{k_{[i]}-1}\left(\alpha_{[i]} j+(f-i+1) \overline{m \gamma}\right)^{(1 /(\eta+1))}\left(\nu_{[i],[j]} p_{[i],[j]} j^{a_{[i],[j]}}\right)^{(\eta /(\eta+1))} \\
& \quad+\left(\eta^{(1 /(\eta+1))}+\eta^{(-\eta /(\eta+1))}\right) \sum_{i=1}^{f} \sum_{j=k_{[i]}}^{m_{[i]}}\left(\beta_{[i]}\left(m_{[i]}-j\right)\right)^{(1 /(\eta+1))}\left(\nu_{[i],[j]} p_{[i],[j]} j^{a_{[i],[j]}}\right)^{(\eta /(\eta+1))} .
\end{aligned}
$$

Let $x_{i, r}=1$, if group $G_{i}$ is assigned to position $r$, and $x_{i, r}=0$ otherwise. For $((f(f+1) \overline{s m \gamma}) / 2)$ is a constant, hence the optimal group sequence $\pi_{G}^{*}$ can be obtained by an assignment problem (AP-G):

$$
\begin{aligned}
& \text { (AP - G }) \text { Min } \sum_{i=1}^{f} \sum_{r=1}^{f} \vartheta_{i, r} x_{i, r} \\
& \text { s.t. } \sum_{r=1}^{f} x_{i, r}=1, \quad i=1,2, \ldots, f, \\
& \sum_{i=1}^{f} x_{i, r}=1, \quad r=1,2, \ldots, f, \\
& x_{i, r}=0 \text { or } 1,
\end{aligned}
$$

where the optimal sequence of group $G_{i}$ is $\pi_{i}^{*}=\left(J_{i,(1)}, J_{i,(2)}\right.$, $\ldots, J_{i,\left(m_{i}\right)}$ ) (by Lemma 4), and

$$
\begin{aligned}
\vartheta_{i, r}= & \left(\eta^{(1 /(\eta+1))}+\eta^{(-\eta /(\eta+1))}\right) \sum_{j=1}^{k_{i}-1}\left(\alpha_{i} j+(f-r+1) \overline{m \gamma}\right)^{(1 /(\eta+1))} \\
& \cdot\left(\nu_{i,(j)} p_{i,(j)} j^{a_{i,(j)}}\right)^{(\eta /(\eta+1))} \\
& +\left(\eta^{(1 /(\eta+1))}+\eta^{(-\eta /(\eta+1))}\right) \sum_{j=k_{i}}^{m_{i}}\left(\beta_{i}\left(m_{i}-j\right)\right)^{(1 /(\eta+1))} \\
& \cdot\left(v_{i,(j)} p_{i,(j)} j^{a_{i,(j)}}\right)^{(\eta /(\eta+1))} .
\end{aligned}
$$

For the problem 1|GT, $s_{i}, \mathrm{CRA}, \mathrm{SLK}, s_{i}=s, m_{i} \gamma_{i}=$ $\overline{m \gamma} \mid \sum_{i=1}^{f} \quad \sum_{j=1}^{m_{i}}\left(\alpha_{i} E_{i, j}+\beta_{i} T_{i, j}+\gamma_{i} q_{i}\right)+\sum_{i=1}^{f} \sum_{j=1}^{m_{i}} v_{i, j} u_{i, j}$, the optimal solution algorithm is given as follows: 
Step 1. Calculate $k_{[i]}$ by using $(4), i=1,2, \ldots, f$.

Step 2. The optimal sequence between groups is arranged in nondecreasing order of $s_{[i]}$.

Step 3. The jobs in each group are arranged according to the assignment problem AP (Lemma 4).

Step 4. Calculate the optimal resource allocation $u_{[i][j]}^{*}$ according to (5).

Step 5. Calculate the optimal common flow allowance $q_{[i]}=C_{[i],\left[k_{[i]}\right]-1}$ and the corresponding optimal objective function

$\sum_{i=1}^{f} \sum_{j=1}^{m_{i}}\left(\alpha_{i} E_{i, j}+\beta_{i} T_{i, j}+\gamma_{i} q_{i}\right)+\sum_{i=1}^{f} \sum_{j=1}^{m_{i}} v_{i, j} u_{i, j}$ by using $(7)$.

Algorithm 1: Optimal solution for Case 1.

Theorem 2. The problem,

$$
\begin{gathered}
1 \mid \mathrm{GT}, s_{i} \text {, CRA, SLK, } s_{i}=s, m_{i} \gamma_{i}=\overline{m \gamma} \mid \sum_{i=1}^{f} \sum_{j=1}^{m_{i}} \\
\cdot\left(\alpha_{i} E_{i, j}+\beta_{i} T_{i, j}+\gamma_{i} q_{i}\right)+\sum_{i=1}^{f} \sum_{j=1}^{m_{i}} v_{i, j} u_{i, j},
\end{gathered}
$$

can be solved by Algorithm 2 in $\mathrm{O}\left(n^{3}\right)$ time.

Proof. Time complexity of Step 1 is $O(n)$; time complexity of Step 2 is $O(f \log f)$ time. Step 3 needs $\sum_{i=1}^{f} O\left(m_{i}^{3}\right) \leq O\left(n^{3}\right)$ time. Steps 4-5 need $O(n)$ time, respectively. Thus, the total time complexity of Algorithm 2 is $O\left(n^{3}\right)$ time.

5.3. Case 3. In this subsection, it is assumed that the number of groups $f$ is a given constant.

Theorem 3. For the $1\left|G T, s_{i}, C R A, S L K\right| \sum_{i=1}^{f} \sum_{j=1}^{m_{i}}\left(\alpha_{i} E_{i, j}+\right.$ $\left.\beta_{i} T_{i, j}+\gamma_{i} q_{i}\right)+\sum_{i=1}^{f} \sum_{j=1}^{m_{i}} v_{i, j} u_{i, j}$ problem, an optimal schedule can be solved in $O\left(f ! n^{3}\right)$ time, i.e., the problem $1\left|G T, s_{i}, C R A, S L K\right| \sum_{i=1}^{f} \sum_{j=1}^{m_{i}}\left(\alpha_{i} E_{i, j}+\beta_{i} T_{i, j}+\gamma_{i} q_{i}\right)+\sum_{i=1}^{f}$ $\sum_{j=1}^{m_{i}} v_{i, j} u_{i, j}$ can be solved in polynomial time if $f$ is a given constant.

Proof. From Lemma 4, if the schedule of groups is given, then an optimal schedule can be obtained in $\sum_{i=1}^{f} O\left(n_{i}^{3}\right) \leq O\left(n^{3}\right)$ time. Obviously, there are $f$ ! possible group schedules, hence an optimal schedule can be solved in $O\left(f ! n^{3}\right)$ time.

Based on Theorem 2, an algorithm can be proposed to solve the problem 1|GT, $s_{i}, \mathrm{CRA}, \mathrm{SLK} \mid \sum_{i=1}^{f} \sum_{j=1}^{m_{i}}\left(\alpha_{i} E_{i, j}+\right.$ $\left.\beta_{i} T_{i, j}+\gamma_{i} q_{i}\right)+\sum_{i=1}^{f} \sum_{j=1}^{m_{i}} v_{i, j} u_{i, j}$.

\section{General Case}

For the general case of the problem 1|GT, $s_{i}$, CRA, SLK| $\sum_{i=1}^{f} \sum_{j=1}^{m_{i}}\left(\alpha_{i} E_{i}, \quad j+\beta_{i} T_{i, j}+\gamma_{i} q_{i}\right)+\sum_{i=1}^{f} \sum_{j=1}^{m_{i}} v_{i, j} u_{i, j}$, the complexity is an open question. Hence, the heuristic algorithm and branch-and-bound $(\mathrm{B} \& \mathrm{~B})$ algorithm might be a good way to solve the problem.

6.1. Heuristic Algorithm. From Lemma 4, the optimal job sequence within the same group can be obtained, and the optimal resource allocation of a given schedule can be obtained by Lemma 3. In this subsection, we apply the wellknown heuristic procedure from the study by Nawaz et al. [46], and the following heuristic algorithm can be proposed.

6.2. Tabu Search Algorithm. Tabu search (TS) algorithm is a metaheuristic algorithm first proposed by Glover [47]. In this subsection, tabu search (TS) is used to find a nearoptimal solution (Xu et al. [48]). The initial sequence used in the TS algorithm is chosen by the nondecreasing order of $s_{i}$, and the maximum number of iterations for the TS algorithm is set at $100 f$, where $f$ is the number of groups. As in the study by $\mathrm{Wu}$ et al. [49], the implementation of the TS algorithm is given as follows:

6.3. A Lower Bound. Let $\pi_{G}=\left[\pi_{G S}, \pi_{G U}\right]$ be a sequence of groups in which $\pi_{G S}$ is the scheduled part, and $\pi_{G U}$ is a unscheduled part. Assume that there are $g$ groups in $\pi_{G S}$; from (7), it is noticed that the terms,

$$
\begin{aligned}
& \sum_{i=g+1}^{f}\left(\sum_{h=i}^{f} m_{[h]} \gamma_{[h]}\right) s_{[i]}, \\
& \cdot\left(\eta^{(1 /(\eta+1))}+\eta^{(-\eta /(\eta+1))}\right) \sum_{i=g+1}^{f} \sum_{j=1}^{k_{[i]}-1}\left(\alpha_{[i]} j+\sum_{h=i}^{f} m_{[h]} \gamma_{[h]}\right)^{(1 /(\eta+1))}\left(\nu_{[i],[j]} p_{[i],[j]} j^{a_{[i],[j]}}\right)^{(\eta /(\eta+1))} \\
& +\left(\eta^{(1 /(\eta+1))}+\eta^{(-\eta /(\eta+1))}\right) \sum_{i=g+1}^{f} \sum_{j=k_{[i]}}^{m_{[i]}}\left(\beta_{[i]}\left(m_{[i]}-j\right)\right)^{(1 /(\eta+1))}\left(\nu_{[i],[j]} p_{[i],[j]} j^{a_{[i],[j]}}\right)^{(\eta /(\eta+1))},
\end{aligned}
$$


Step 1. Calculate $k_{[i]}$ by using $(4), i=1,2, \ldots, f$.

Step 2. The optimal sequence between groups can be obtained by Lemma 8 .

Step 3. The jobs in each group are arranged according to the assignment problem AP (Lemma 4).

Step 4.Calculate the optimal resource allocation $u_{[i],[j]}^{*}$ according to (5).

Step 5. Calculate the optimal common flow allowance $q_{[i]}=C_{[i]\left[k_{[i]}\right]-1}$ and the corresponding optimal objective function $\sum_{i=1}^{f} \sum_{j=1}^{m_{i}}\left(\alpha_{i} E_{i, j}+\beta_{i} T_{i, j}+\gamma_{i} q_{i}\right)+\sum_{i=1}^{f} \sum_{j=1}^{m_{i}} v_{i, j} u_{i, j}$ by using (7).

Algorithm 2: Optimal solution for Case 2.

can be minimized by Lemmas $5-6$. Hence, the lower bound can be obtained by the following formula:

$$
\begin{aligned}
& L B=\sum_{i=1}^{g}\left(\sum_{h=i}^{g} m_{[h]} \gamma_{[h]}+\sum_{h=g+1}^{f} m_{\langle h\rangle} \gamma_{\langle h\rangle}\right) s_{[i]}+\sum_{i=g+1}^{f}\left(\sum_{h=i}^{f} m_{\langle h\rangle} \gamma_{\langle h\rangle}\right) s_{(i)} \\
& +\left(\eta^{(1 /(\eta+1))}+\eta^{(-\eta /(\eta+1))}\right)\left(\begin{array}{c}
\sum_{i=1}^{g} \sum_{j=1}^{k_{[i]}-1}\left(\alpha_{[i]} j+\sum_{h=i}^{g} m_{[h]} \gamma_{[h]}+\sum_{h=g+1}^{f} m_{\langle h\rangle} \gamma_{\langle h\rangle}\right)^{(1 /(\eta+1))} \\
\times\left(\nu_{[i],[j]} p_{[i],[j]} j^{a_{[i],[j]}}\right)^{(\eta /(\eta+1))} \\
+\sum_{i=g+1}^{f} \sum_{j=1}^{k_{[i]}-1}\left(\alpha_{[i]} j+\sum_{h=i}^{g} m_{[h]} \gamma_{[h]}+\sum_{h=g+1}^{f} m_{\langle h\rangle} \gamma_{\langle h\rangle}\right)^{(1 /(\eta+1))} \\
\times\left(\nu_{[i],[j]} p_{[i],[j]} j^{a_{[i],[j]}}\right)^{(\eta /(\eta+1))}
\end{array}\right) \\
& +\left(\eta^{(1 /(\eta+1))}+\eta^{(-\eta /(\eta+1))}\right)\left(\begin{array}{c}
\sum_{i=1}^{g} \sum_{j=k_{[i]}}^{m_{[i]}}\left(\beta_{[i]}\left(m_{[i]}-j\right)\right)^{(1 /(\eta+1))}\left(\nu_{[i],[j]} p_{[i],[j]} j^{a_{[i],[j]}}\right)^{(\eta /(\eta+1))} \\
+\sum_{i=g+1}^{f} \sum_{j=k_{[i]}}^{m_{[i]}}\left(\beta_{[i]}\left(m_{[i]}-j\right)\right)^{(1 /(\eta+1))}\left(\nu_{[i],[j]} p_{[i],[j]} j^{a_{[i],[j]}}\right)^{(\eta /(\eta+1))}
\end{array}\right),
\end{aligned}
$$

where $s_{(g+1)} \leq s_{(g+2)} \leq \cdots \leq s_{(f)}$ and $m_{\langle g+1\rangle} \gamma_{\langle g+1\rangle} \geq m_{\langle g+2\rangle}$ $\gamma_{\langle g+2\rangle} \geq \cdots \geq m_{\langle f\rangle} \gamma_{\langle f\rangle}$ (note that $s_{(i)}$ and $m_{\langle i\rangle} \gamma_{\langle i\rangle}((i=g+$ $1, r+2, \ldots, f))$ do not necessarily correspond to the same group).

6.4. Branch-and-Bound (B\&B) Algorithm. The branchand-bound $(B \& B)$ algorithm search follows a depth-first strategy; this algorithm assigns groups in a forward manner starting from the first position (assign a group to a node).

6.5. An Example for B\&B Algorithm. Consider an example in which there are 13 jobs belonging to 5 groups $G_{1}=\left\{J_{11}, J_{12}\right\}$,
$G_{2}=\left\{J_{21}, J_{22}, J_{23}\right\}, G_{3}=\left\{J_{31}, J_{32}, J_{33}\right\}, G_{4}=\left\{J_{41}, J_{42}\right\}$, and $G_{5}=\left\{J_{51}, J_{52}, J_{53}\right\}$. The processing times of each job, learning rate, and setup time of each group and other parameters are shown in Tables 1 and 2 .

From Algorithm 3 (HA), the initial sequence is $\left[G_{1}, G_{2}, G_{4}, G_{5}, G_{3}\right]$, and the objective function value (upper bound) is $\sum_{i=1}^{f} \sum_{j=1}^{m_{i}}\left(\alpha_{i} E_{i, j}+\beta_{i} T_{i, j}+\gamma_{i} q_{i}\right)+\sum_{i=1}^{f} \sum_{j=1}^{m_{i}} v_{i, j}$ $u_{i, j}=295.8274$. According to Algorithm 4 (B\&B algorithm), the following search tree can be obtained, which is represented by Figure 1. The numbers in Figure 1 represent the lower bound values, and $G_{0}$ is defined as the level 0 .

At level 1, i.e., $g=1$, for group $G_{1}$, from formula (8), the lower bound is 
TABLE 1: Numerical parameters.

\begin{tabular}{llllcc}
\hline & $s_{i}$ & $\alpha_{i}$ & $\beta_{i}$ & $\gamma_{i}$ & $u_{i}^{\prime}$ \\
\hline$G_{1}$ & 3 & 1 & 2 & 1 & 1 \\
$G_{2}$ & 5 & 1 & 2 & 1 & 1 \\
$G_{3}$ & 7 & 1 & 2 & 1 & 1 \\
$G_{4}$ & 4 & 1 & 2 & 1 & 1 \\
$G_{5}$ & 6 & 1 & 2 & 1 & 1 \\
\hline
\end{tabular}

TABLE 2: Numerical parameters.

\begin{tabular}{|c|c|c|c|c|c|c|c|c|c|c|c|c|c|}
\hline & \multicolumn{13}{|c|}{$\eta=1$} \\
\hline & $J_{11}$ & $J_{12}$ & $J_{21}$ & $J_{22}$ & $J_{23}$ & $J_{31}$ & $J_{32}$ & $J_{33}$ & $J_{41}$ & $J_{42}$ & $J_{51}$ & $J_{52}$ & $J_{53}$ \\
\hline$p_{i, j}$ & 4 & 6 & 5 & 3 & 5 & 4 & 7 & 7 & 6 & 3 & 2 & 8 & 5 \\
\hline$a_{i, j}$ & -0.16 & -0.21 & -0.22 & -0.12 & -0.24 & -0.23 & -0.32 & -0.31 & -0.15 & -0.17 & -0.19 & -0.2 & -0.26 \\
\hline$v_{i, j}$ & 5 & 4 & 7 & 6 & 8 & 6 & 3 & 4 & 2 & 5 & 6 & 4 & 5 \\
\hline
\end{tabular}

(1) Input: $k_{[i]} \longleftarrow$ using equation (4) for $i=1,2, \ldots, f$; the internal job sequence $\pi_{i}^{*} \longleftarrow$ using Lemma 4 for each group $G_{i}$, $i=1,2, \ldots, f$.

(2) Output: the suboptimal resource allocation $u_{[i],[j]}^{*}$, suboptimal common flow allowance $q_{[i]}=, C_{[i]\left[k_{[i]}\right]-1}$, and corresponding objective function value $\sum_{i=1}^{f} \sum_{j=1}^{m_{i}}\left(\alpha_{i} E_{i, j}+\beta_{i} T_{i, j}+\gamma_{i} q_{i}\right)+, \sum_{i=1}^{f} \sum_{j=1}^{m_{i}} v_{i, j} u_{i, j}$.

3) For each $\pi_{i}^{*}$, groups are scheduled by the nondecreasing order of $s_{i},(i=1,2, \ldots, f)$;

(4) For each $\pi_{i}^{*}$, groups are scheduled by the nonincreasing order of $m_{i} \gamma_{i},(i=1,2, \ldots, f)$;

(5) From Step 3 and Step 4 , the smallest objective function value $\sum_{i=1}^{f} \sum_{j=1}^{m_{i}}\left(\alpha_{i} E_{i, j}+\beta_{i} T_{i, j}+\gamma_{i} q_{i}\right),+\sum_{i=1}^{f} \sum_{j=1}^{m_{i}} v_{i, j} u_{i, j}$ is selected as the original group sequence $\pi_{G}$;

(6) Pick the two groups from the first and second position of the list of Step 5 and find the best sequence for these two groups by calculating $\sum_{i=1}^{f} \sum_{j=1}^{m_{i}}\left(\alpha_{i} E_{i, j}+\beta_{i} T_{i, j}+\gamma_{i} q_{i}\right)+\sum_{i=1}^{f} \sum_{j=1}^{m_{i}} v_{i, j} u_{i, j}$ for the two possible sequences. Do not change the relative positions of these two groups with respect to each other in the remaining steps of the algorithm. Set $i=3$;

(7) Pick the group in the $i$ th position of the list generated in Step 5 and find the best sequence by placing it at all possible $i$ positions in the partial sequence found in the previous step, without changing the relative positions to each other of the already assigned groups. The number of enumerations at this step equals $i$;

(8) If $i>f$, then stop

(9) Otherwise,

(10) $i \longleftarrow i+1$, and return to Step 7;

(11) Calculate the suboptimal resource allocation $u_{[i],[j]}^{*}$ according to (5). Calculate the suboptimal common flow allowance $q_{[i]}=$ $C_{[i]\left[k_{[i]}\right]-1}$ and the corresponding optimal objective function $\sum_{i=1}^{f} \sum_{j=1}^{m_{i}}\left(\alpha_{i} E_{i, j}+\beta_{i} T_{i, j}+\gamma_{i} q_{i}\right)+\sum_{i=1}^{f} \sum_{j=1}^{m_{i}} v_{i, j} u_{i, j}$ by using (7).

Algorithm 3: HA.

Step 1. Finding the upper bound: use Algorithm 3 to obtain an initial solution for the problem.

Step 2. Bounding: calculate the lower bound (see (8)) for the node. If the lower bound for an unfathomed partial schedule of groups is larger than or equal to the value of the objective function of the initial solution, eliminate the node and all the nodes following it in the branch. Calculate the objective function value of the completed schedule; if it is less than the initial solution, replace it as the new solution; otherwise, eliminate it.

Step 3. Termination: continue until all nodes have been explored. 


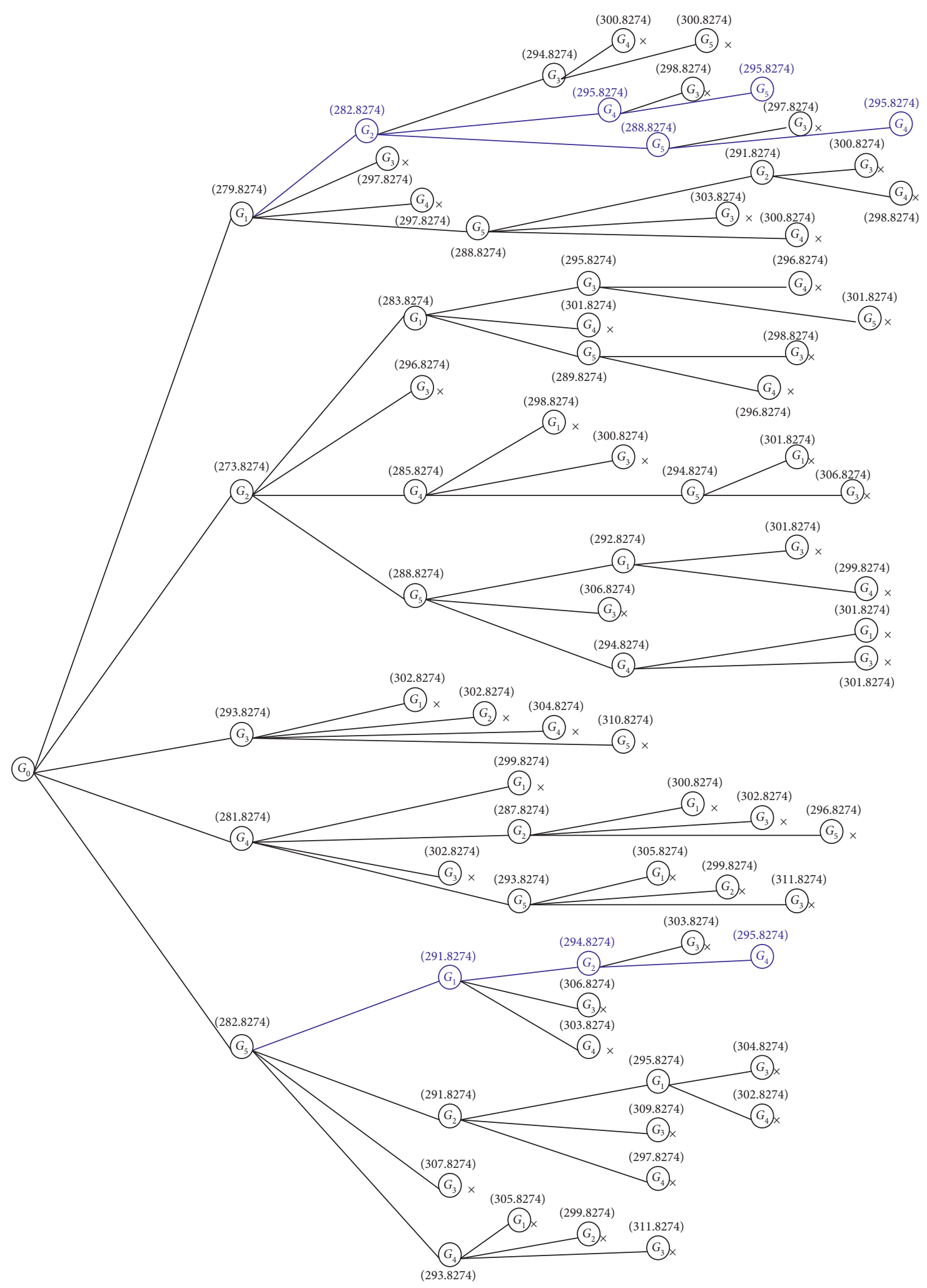

Figure 1: Search tree of the B\&B algorithm for the example ( $\times$ denotes pruning). 
Step 1. Calculate $k_{[i]}$ by using $(4), i=1,2, \ldots, f$.

Step 2. The jobs in each group are arranged according to the assignment problem AP (Lemma 4).

Step 3. List all the group schedules.

Step 4. For each group schedule, calculate the corresponding objective value $\sum_{i=1}^{f} \sum_{j=1}^{m_{i}}\left(\alpha_{i} E_{i, j}+\beta_{i} T_{i, j}+\gamma_{i} q_{i}\right)+\sum_{i=1}^{f} \sum_{j=1}^{m_{i}} v_{i, j} u_{i, j}$.

Step 5. Comparing all the objective values $\sum_{i=1}^{f} \sum_{j=1}^{m_{i}}\left(\alpha_{i} E_{i, j}+\beta_{i} T_{i, j}+\gamma_{i} q_{i}\right)+\sum_{i=1}^{f} \sum_{j=1}^{m_{i}} v_{i, j} u_{i, j}$, the minimum one is optimal, and its corresponding schedule is the optimal sequence of the problem $1 \mid \mathrm{GT}, s_{i}$, CRA, SLK| $\sum_{i=1}^{f} \sum_{j=1}^{m_{i}}\left(\alpha_{i} E_{i, j}+\beta_{i} T_{i, j}+\gamma_{i} q_{i}\right)+\sum_{i=1}^{f} \sum_{j=1}^{m_{i}} v_{i, j} u_{i, j}$.

Algorithm 5: Optimal solution for Case 3.

Step 1. Let the tabu list be empty and the iteration number be zero.

Step 2. Set the initial sequence of the TS algorithm, calculate its objective function, and set the current sequence as the best solution $\pi^{*}$.

Step 3. Search the associated neighborhood of the current sequence and resolve if there is a sequence $\pi^{* *}$ with the smallest objective function in associated neighborhood and it is not in the tabu list.

Step 4. If $\pi^{* *}$ is better than $\pi^{*}$, then let $\pi^{*}=\pi^{* *}$. Update the tabu list and the iteration number.

Step 5. If there is not a sequence in associated neighborhood but it is not in the tabu list or the maximum number of iterations is reached, then output the final sequence. Otherwise, update tabu list and go to Step 3.

Algorithm 6: TS.

$$
\begin{aligned}
& L B\left(G_{1}\right)=(2 \times 1+3 \times 1+3 \times 1+3 \times 1+2 \times 1) \times 3 \\
&+(3 \times 1+3 \times 1+3 \times 1+2 \times 1) \times 4+(3 \times 1+3 \times 1+2 \times 1) \times 5 \\
&+(3 \times 1+2 \times 1) \times 6+2 \times 1 \times 7 \\
&+2 \times\left(\begin{array}{l}
(2 \times(2-1))^{(1 / 2)} \times(4 \times 5)^{(1 / 2)} \\
+(2 \times(3-1))^{(1 / 2)} \times(3 \times 6)^{(1 / 2)}+(2 \times(3-2))^{(1 / 2)} \times\left(5 \times 7 \times 2^{-0.22}\right)^{(1 / 2)} \\
+(2 \times(3-1))^{(1 / 2)} \times(3 \times 7)^{(1 / 2)}+(2 \times(3-2))^{(1 / 2)} \times\left(6 \times 4 \times 2^{-0.23}\right)^{(1 / 2)} \\
+(2 \times(3-1))^{(1 / 2)} \times(6 \times 2)^{(1 / 2)}+(2 \times(3-2))^{(1 / 2)} \times\left(5 \times 5 \times 2^{-0.26}\right)^{(1 / 2)} \\
+(2 \times(2-1))^{(1 / 2)} \times(2 \times 6)^{(1 / 2)}
\end{array}\right)
\end{aligned}
$$

$=279.8274$.

The calculation process of lower bounds of the remaining node is similar to that of this node. From Figure 1, the optimal sequences are $\left[G_{1}, G_{2}, G_{4}, G_{5}, G_{3}\right],\left[G_{1}, G_{2}, G_{5}, G_{4}, G_{3}\right]$, and $\left[G_{5}, G_{1}, G_{2}, G_{4}, G_{3}\right]$, and the optimal value of objective function is $\sum_{i=1}^{f} \sum_{j=1}^{m_{i}}\left(\alpha_{i} E_{i, j}+\beta_{i} T_{i, j}+\gamma_{i} q_{i}\right)+\sum_{i=1}^{f} \sum_{j=1}^{m_{i}} v_{i, j} u_{i, j}$ $=295.8274$.

6.6. Computational Experiments. An enumeration algorithm (i.e., Algorithm 5), heuristic algorithm (i.e., Algorithm 3), TS algorithm (i.e., Algorithm 6), and B\&B algorithm (i.e., Algorithm 4) were programmed in $\mathrm{C}++$ and carried out on a CPU Intel Core i5-8250U $1.4 \mathrm{GHz}$ PC with $8.00 \mathrm{~GB}$ RAM. The number of jobs and groups $n=50,100,150,200$ and $f=8,9,10,11,12$ were tested, and each group must contain at least one job. The parameters setting can be obtained as follows: $s_{i}, v_{i, j}, \alpha_{i}, \beta_{i}$, and $\gamma_{i}$ were drawn from a discrete uniform distribution in $[1,10] ; p_{i, j}$ were drawn from a discrete uniform distribution in $[1$,
100]; $a_{i, j}$ were drawn from a uniform distribution in $[-0.1$, $-1]$; and $\eta=2$. To avoid the contingency, each problem instance was conducted 20 times, setting the maximum CPU time per instance at 3600 seconds. The percentage relative error of the solution produced by Algorithms 3 and 6 is calculated as

$$
\frac{Z(A i)-Z^{*}}{Z^{*}} \times 100 \%,
$$

where $i=4,5, Z(A i)$, and $Z^{*}$ are the objective function values $\sum_{i=1}^{f} \sum_{j=1}^{m_{i}}\left(\alpha_{i} E_{i, j}+\beta_{i} T_{i, j}+\gamma_{i} q_{i}\right)+\sum_{i=1}^{f} \sum_{j=1}^{m_{i}} v_{i, j} u_{i, j}$ generated by Algorithm 1 and Algorithm 4, respectively.

On the other hand, "A3-CPU time (s)" ("A4-CPU time (s)," "A5-CPU time (s)," and "A6-(B\&B) CPU time (s)") as the running time of Algorithm 5 (Algorithms 3-4) is defined. The results are summarized in Table 3. From Table 3, it is easy to find that the maximum relative error percentage of Algorithm 3 is less than $6.1 \%$ for $n \leq 200$, and performance of Algorithm 3 (i.e., the heuristic algorithm) performs very well in terms of the 
TABle 3: Results of Algorithms.

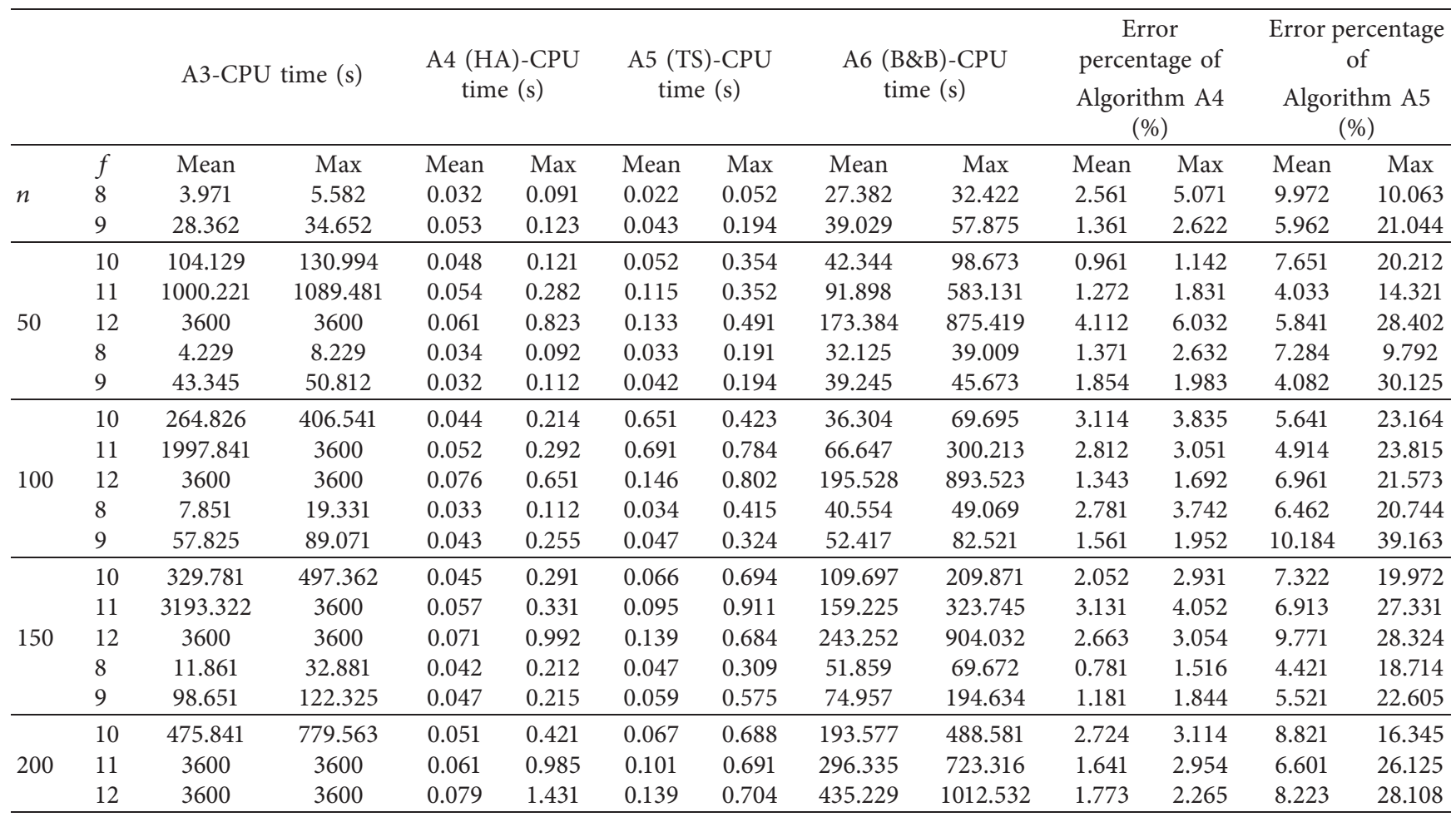

relative error percentages. Moreover, Table 3 shows that the mean CPU time (s) for the enumeration algorithm (i.e., Algorithm 5) is larger than the B\&B algorithm (i.e., Algorithm 4).

\section{Conclusions}

In this paper, we studied the single-machine resource allocation scheduling problem with learning effect and group technology. The goal is to determine the optimal sequence of jobs and groups, the optimal common flow allowances, and the optimal resource allocation such that the weighted sum of the scheduling cost and the resource allocation cost is minimized. For some special cases (i.e., cases $\left(s_{i} \leq s_{h}\right) \Longrightarrow\left(m_{i} \gamma_{i} \geq m_{h} \gamma_{h}\right), s_{i}=s, m_{i} \gamma_{i}=\overline{m \gamma}$, and $f$ is a given constant), it was shown that the problem can be solved in polynomial time. For the general case of the problem, the heuristic, tabu search, and B\&B algorithms were proposed. The results show that the maximum relative percentage error of the proposed heuristic algorithm (i.e., Algorithm 3) from optimal solutions is less than $6.1 \%$ for all sizes of instances.

Further research may focus on the extensions of this model to more complicated machine setting (such as flow shop and/or parallel machines) or study other nonregular objective functions (such as due-window assignment scheduling problems with position-dependent weights, see the study by Wang et al. [50]).

\section{Data Availability}

The data used to support the findings of this study are available from the corresponding author upon reasonable request.

\section{Conflicts of Interest}

The authors declare that they have no conflicts of interest.

\section{Acknowledgments}

This research was supported by the National Natural Science Foundation of China (71903020 and 71672019) and the Natural Science Foundation of Liaoning Province, China (20180550743). Ji-Bo Wang was also supported by the Natural Science Foundation of Liaoning Province, China (2020-MS-233).

\section{References}

[1] D. Shabtay and G. Steiner, "A survey of scheduling with controllable processing times," Discrete Applied Mathematics, vol. 155, no. 13, pp. 1643-1666, 2007.

[2] D. Biskup, "A state-of-the-art review on scheduling with learning effects," European Journal of Operational Research, vol. 188, no. 2, pp. 315-329, 2008.

[3] A. Azzouz, M. Ennigrou, and L. Ben Said, "Scheduling problems under learning effects: classification and cartography," International Journal of Production Research, vol. 56, no. 4, pp. 1642-1661, 2018.

[4] Z. Zhu, L. Sun, F. Chu, and M. Liu, "Single-machine group scheduling with resource allocation and learning effect," Computers \& Industrial Engineering, vol. 60, no. 1, pp. 148157, 2011.

[5] Y.-Y. Lu, J.-B. Wang, P. Ji, and H. He, "A note on resource allocation scheduling with group technology and learning effects on a single machine," Engineering Optimization, vol. 49, no. 9, pp. 1621-1632, 2017. 
[6] L. Sun, A. J. F. Yu, and B. Wu, "Single machine common flow allowance group scheduling with learning effect and resource allocation," Computers and Industrial Engineering, vol. 139, Article ID 106126, 2020.

[7] D.-Y. Lv, S.-W. Luo, J. Xue, J.-X. Xu, and J.-B. Wang, “A note on single machine common flow allowance group scheduling with learning effect and resource allocation," Computers \& Industrial Engineering, vol. 151, Article ID 106941, 2021.

[8] J.-B. Wang, J. Xu, and J. Yang, "Bi-criterion optimization for flow shop with a learning effect subject to release dates," Complexity, vol. 2018, Article ID 9149510, 12 pages, 2018.

[9] J. B. Wang, F. Liu, and J. J. Wang, "Research on $\mathrm{m}$-machine flow shop scheduling with truncated learning effects $m$-machine flow shop scheduling with truncated learning effects," International Transactions in Operational Research, vol. 26, no. 3, pp. 11351151, 2019.

[10] P. Yan, J.-B. Wang, and L.-Q. Zhao, "Single-machine bicriterion scheduling with release times and exponentially time-dependent learning effects," Journal of Industrial and Management Optimization, vol. 15, no. 3, pp. 1117-1131, 2019.

[11] J.-B. Wang, M. Gao, J.-J. Wang, L. Liu, and H. He, "Scheduling with a position-weighted learning effect and job release dates," Engineering Optimization, vol. 52, no. 9, pp. 1475-1493, 2020.

[12] X. Sun, X.-N. Geng, and F. Liu, "Flow shop scheduling with general position weighted learning effects to minimise total weighted completion time," Journal of the Operational Research Society, 2020.

[13] V. Kayvanfar, I. Mahdavi, and G. M. Komaki, "Single machine scheduling with controllable processing times to minimize total tardiness and earliness," Computers \& Industrial Engineering, vol. 65, no. 1, pp. 166-175, 2013.

[14] V. Kayvanfar, I. Mahdavi, and G. H. M. Komaki, "A drastic hybrid heuristic algorithm to approach to JIT policy considering controllable processing times," International Journal of Advanced Manufacturing Technology, vol. 69, no. 1-4, pp. 257-267, 2013.

[15] Y.-Y. Lu and J.-Y. Liu, "A note on resource allocation scheduling with position-dependent workloads," Engineering Optimization, vol. 50, no. 10, pp. 1810-1827, 2018.

[16] Y.-C. Tsao, V.-V. Thanh, and F.-J. Hwang, "Energy-efficient single-machine scheduling problem with controllable job processing times under differential electricity pricing," Resources," Conservation and Recycling, vol. 161, Article ID 104902, 2020.

[17] B. Mor, D. Shabtay, and L. Yedidsion, "Heuristic algorithms for solving a set of NP-hard single-machine scheduling problems with resource-dependent processing times," Computers \& Industrial Engineering, vol. 153, Article ID 107024, 2021.

[18] V. Kayvanfar, G. M. Komaki, A. Aalaei, and M. Zandieh, "Minimizing total tardiness and earliness on unrelated parallel machines with controllable processing times," Computers \& Operations Research, vol. 41, pp. 31-43, 2014.

[19] M. H. F. Zarandi and V. Kayvanfar, "A bi-objective identical parallel machine scheduling problem with controllable processing times: a just-in-time approach," International Journal of Advanced Manufacturing Technology, vol. 77, no. 1-4, pp. 545-563, 2015.

[20] V. Kayvanfar, M. Zandieh, and E. Teymourian, “An intelligent water drop algorithm to identical parallel machine scheduling with controllable processing times: a just-in-time approach," Computational and Applied Mathematics, vol. 36, no. 1, pp. 159-184, 2017.
[21] C. T. Mosier, D. A. Elvers, and D. Kelly, "Analysis of group technology scheduling heuristics," International Journal of Production Research, vol. 22, no. 5, pp. 857-875, 1984.

[22] S. Webster and K. R. Baker, "Scheduling groups of jobs on a single machine," Operations Research, vol. 43, no. 4, pp. 692-703, 1995.

[23] S.-J. Yang and D.-L. Yang, "Single-machine scheduling simultaneous with position-based and sum-of-processing-times-based learning considerations under group technology assumption," Applied Mathematical Modelling, vol. 35, no. 5, pp. 2068-2074, 2011.

[24] M. Ji, K. Chen, J. Ge, and T. C. E. Cheng, "Group scheduling and job-dependent due window assignment based on a common flow allowance," Computers \& Industrial Engineering, vol. 68, pp. 35-41, 2014.

[25] Y.-T. Xu, Y. Zhang, and X. Huang, "Single-machine ready times scheduling with group technology and proportional linear deterioration," Applied Mathematical Modelling, vol. 38, no. 1, pp. 384-391, 2014.

[26] F. Liu, J. Yang, and Y.-Y. Lu, "Solution algorithms for singlemachine group scheduling with ready times and deteriorating jobs," Engineering Optimization, vol. 51, no. 5, pp. 862-874, 2019.

[27] W.-X. Li and C.-L. Zhao, "Single machine scheduling problem with multiple due windows assignment in a group technology," Journal of Applied Mathematics and Computing, vol. 48, no. 1-2, pp. 477-494, 2015.

[28] X. Zhang and Q. Xie, "Single machine group scheduling with position dependent processing times and ready times," Mathematical Problems in Engineering, vol. 2015, Article ID 206230, 9 pages, 2015.

[29] P. Ji and L. Li, "Single-machine group scheduling problems with variable job processing times," Mathematical Problems in Engineering, vol. 2015, Article ID 758919, 9 pages, 2015.

[30] X. Zhang, L. Liao, W. Zhang, T. C. E. Cheng, Y. Tan, and M. Ji, "Single-machine group scheduling with new models of position-dependent processing times," Computers \& Industrial Engineering, vol. 117, pp. 1-5, 2018.

[31] S. Muştu and T. Eren, "The single machine scheduling problem with setup times under an extension of the general learning and forgetting effects," Optimization Letters, 2020.

[32] A. Allahverdi, "The third comprehensive survey on scheduling problems with setup times/costs," European Journal of Operational Research, vol. 246, no. 2, pp. 345-378, 2015.

[33] J. S. Neufeld, J. N. D. Gupta, and U. Buscher, "A comprehensive review of flowshop group scheduling literature," Computers \& Operations Research, vol. 70, pp. 56-74, 2016.

[34] D. Wang, M.-Z. Wang, and J.-B. Wang, "Single-machine scheduling with learning effect and resource-dependent processing times," Computers \& Industrial Engineering, vol. 59, no. 3, pp. 458-462, 2010.

[35] Y.-Y. Lu, G. Li, Y.-B. Wu, and P. Ji, “Optimal due-date assignment problem with learning effect and resource-dependent processing times," Optimization Letters, vol. 8, no. 1, pp. 113-127, 2014.

[36] H. He, M. Liu, and J.-B. Wang, "Resource constrained scheduling with general truncated job-dependent learning effect," Journal of Combinatorial Optimization, vol. 33, no. 2, pp. 626-644, 2017.

[37] L. Li, P. Yan, P. Ji, and J.-B. Wang, "Scheduling jobs with simultaneous considerations of controllable processing times and learning effect," Neural Computing and Applications, vol. 29, no. 11, pp. 1155-1162, 2018.

[38] J. B. Wang, D. Y. Lv, J. Xu, P. Ji, and F. Li, "Bicriterion scheduling with truncated learning effects and convex 
controllable processing times," International Transactions in Operational Research, vol. 28, no. 3, pp. 1573-1593, 2021.

[39] X.-N. Geng, J.-B. Wang, and D. Bai, "Common due date assignment scheduling for a no-wait flowshop with convex resource allocation and learning effect," Engineering Optimization, vol. 51, no. 8, pp. 1301-1323, 2019.

[40] X. Sun, X.-N. Geng, J.-B. Wang, and F. Liu, "Convex resource allocation scheduling in the no-wait flowshop with common flow allowance and learning effect," International Journal of Production Research, vol. 57, no. 6, pp. 1873-1891, 2019.

[41] W. Liu and C. Jiang, "Due-date assignment scheduling involving job-dependent learning effects and convex resource allocation," Engineering Optimization, vol. 52, no. 1, pp. 74-89, 2020.

[42] J.-B. Wang and X.-X. Liang, "Group scheduling with deteriorating jobs and allotted resource under limited resource availability constraint," Engineering Optimization, vol. 51, no. 2, pp. 231-246, 2019.

[43] X.-X. Liang, M. Liu, Y.-B. Feng, J.-B. Wang, and L.-S. Wen, "Solution algorithms for single-machine resource allocation scheduling with deteriorating jobs and group technology," Engineering Optimization, vol. 52, no. 7, pp. 1184-1197, 2020.

[44] B. Liao, X. Wang, X. Zhu, S. Yang, and P. M. Pardalos, "Less is more approach for competing groups scheduling with different learning effects," Journal of Combinatorial Optimization, vol. 39, no. 1, pp. 33-54, 2020.

[45] G. I. Adamopoulos and C. P. Pappis, "Single machine scheduling with flow allowances," Journal of the Operational Research Society, vol. 47, no. 10, pp. 1280-1285, 1996.

[46] M. Nawaz, E. E. Enscore, and I. Ham, "A heuristic algorithm for the m-machine, $\mathrm{n}$-job flow-shop sequencing problem $m n$ machine,-job flow-shop sequencing problem," Omega, vol. 11, no. 1, pp. 91-95, 1983.

[47] F. Glover, "Tabu search-Part I," ORSA Journal on Computing, vol. 1, no. 3, pp. 190-206, 1989.

[48] K. Xu, Z. Feng, and K. Jun, "A tabu-search algorithm for scheduling jobs with controllable processing times on a single machine to meet due-dates," Computers \& Operations Research, vol. 37, no. 11, pp. 1924-1938, 2010.

[49] C.-C. Wu, W.-H. Wu, W.-H. Wu, P.-H. Hsu, Y. Yin, and J. $\mathrm{Xu}$, "A single-machine scheduling with a truncated linear deterioration and ready times," Information Sciences, vol. 256, pp. 109-125, 2014.

[50] J.-B. Wang, B. Zhang, L. Li, D. Bai, and Y.-B. Feng, "Due-window assignment scheduling problems with position-dependent weights on a single machine," Engineering Optimization, vol. 52, no. 2, pp. 185-193, 2020. 